\title{
Tracking Performance in Ultrasound Super-Resolution Imaging
}

Taghavi, Iman; Andersen, Sofie Bech; Hoyos, Carlos A. Villagomez ; Schou, Mikkel; Øygard, Sigrid Husebø; Gran, Fredrik; Hansen, Kristoffer L.; Sørensen, Charlotte M.; Bachmann Nielsen, Michael; Stuart, Matthias Bo

Total number of authors:

11

Published in:

2020 IEEE International Ultrasonics Symposium

Link to article, DOI:

10.1109/IUS46767.2020.9251605

Publication date:

2020

Document Version

Peer reviewed version

Link back to DTU Orbit

Citation (APA):

Taghavi, I., Andersen, S. B., Hoyos, C. A. V., Schou, M., Øygard, S. H., Gran, F., Hansen, K. L., Sørensen, C. M., Bachmann Nielsen, M., Stuart, M. B., \& Jensen, J. A. (2020). Tracking Performance in Ultrasound SuperResolution Imaging. In 2020 IEEE International Ultrasonics Symposium IEEE. https://doi.org/10.1109/IUS46767.2020.9251605

\section{General rights}

Copyright and moral rights for the publications made accessible in the public portal are retained by the authors and/or other copyright owners and it is a condition of accessing publications that users recognise and abide by the legal requirements associated with these rights.

- Users may download and print one copy of any publication from the public portal for the purpose of private study or research.

- You may not further distribute the material or use it for any profit-making activity or commercial gain

- You may freely distribute the URL identifying the publication in the public portal 


\title{
Tracking Performance in Ultrasound Super-Resolution Imaging
}

\author{
Iman Taghavi ${ }^{1}$, Sofie B. Andersen ${ }^{2,3}$, Carlos A. Villagomez Hoyos ${ }^{4}$, Mikkel Schou ${ }^{1}$, Sigrid H. Øygard ${ }^{1}$, \\ Fredrik Gran ${ }^{4}$, Kristoffer L. Hansen ${ }^{2}$, Charlotte M. Sørensen ${ }^{3}$, Michael B. Nielsen², \\ Matthias B. Stuart ${ }^{1}$, Jørgen Arendt Jensen ${ }^{1}$ \\ ${ }^{1}$ Department of Health Technology, Technical University of Denmark \\ ${ }^{2}$ Department of Diagnostic Radiology, Rigshospital, Denmark \\ ${ }^{3}$ Department of Biomedical Sciences, University of Copenhagen \\ ${ }^{4}$ BK Medical, Herlev, Denmark
}

\begin{abstract}
Tracking plays an important role in super-resolution (SR) ultrasound imaging, as it improves the quality and sharpness of the final SR images. Moreover, tracking enables quantification of clinically important parameters, such as blood flow velocity. However, the tracking performance degrades in the presence of complex particle patterns and localization uncertainty due to noise and motion. This work presents and discusses multiple approaches for tracking evaluation and compares a nearestneighbor (NN) with a Kalman tracker through simulations and an in vivo experiment. It is shown that in the presence of a localization uncertainty with a standard deviation (SD) of $\lambda / 5$, the bias and SD of the velocity estimates reach $-1.04 \pm 0.9$ $\mathrm{mm} / \mathrm{s}$ and $-0.12 \pm 0.72 \mathrm{~mm} / \mathrm{s}$ in the $\mathrm{NN}$ and Kalman tracker, respectively (relative to the peak velocity of $10 \mathrm{~mm} / \mathrm{s}$ ). The precision of individual track positions is estimated for an in vivo experiment as $37.95 \pm 21.37 \mu \mathrm{m}$ and $23.9 \pm 11.82 \mu \mathrm{m}$ for the $\mathrm{NN}$ and Kalman trackers, respectively. The results indicate that the Kalman tracker achieves a better velocity estimation and reduces localization uncertainty.
\end{abstract}

\section{INTRODUCTION}

Ultrasound super-resolution imaging (SRI) has been studied by various research groups over the last years [1]-[7]. The approach employs detection and tracking of microbubble (MB) contrast agents to visualize the micro-vasculature including the capillary network. A detector estimates the centroids of the MBs, and several factors including the non-linear imaging scheme, the signal to noise ratio (SNR), complex and nonuniform $\mathrm{MB}$ concentrations, and various $\mathrm{MB}$ dynamics affect the centroid estimation and tracking of the MB positions. The centroid localization is uncertain, since the above mentioned factors produce non-symmetrical, overlapped, dissimilar, and shifted target spread functions for the various MBs during the scan. These conditions are even worse for in vivo measurements, as several other ultrasound artifacts, organ motion, and uncontrolled $\mathrm{MB}$ concentrations depending on the 3D vessel structure, affect the images.

The tracking methods aim to link the detected MBs from frame to frame. The methods range from simple nearestneighbor (NN) [8] to multi-frame data structure and using explicit motion models (e.g. Kalman filtering) [6], [9]-[11].
This work investigates several performance metrics for tracking evaluation. A NN and a Kalman tracker are compared through both simulations with known ground-truth and in an in vivo experiment with unknown ground-truth. Finally, a new tracking strategy that will likely address tracking difficulties in a wide range of scenarios is suggested.

\section{Methods}

\section{A. Simulation}

The simulations were made by generating MB positions, each with uniformly random lifetime and known groundtruth, moving with different velocities across an X-shaped phantom. A random localization error was added to each MB position. The various parameters of the phantom are listed in Table I. The ground-truth tracks with different MB density and trajectories of MBs with localization uncertainty are shown in Fig. 1. These simulations provided a wide range of scenarios, including various MB concentrations, velocities, and localization uncertainties.

TABLE I: Parameters used in the in-silicon X-phantom

\begin{tabular}{|l|c|}
\hline Parameters & Value \\
\hline Tube length & $10 \mathrm{~mm}$ \\
Tube radius & $250 \mu \mathrm{m} \& 125 \mu \mathrm{m}$ \\
Peak velocity & $10 \mathrm{~mm} / \mathrm{s} \& 5 \mathrm{~mm} / \mathrm{s}$ \\
Velocity profile & Parabolic \\
Angle between tubes & $60^{\circ}$ \\
Point Spread Function (PSF) & Unsymmetrical Gaussian \\
Axial / Lateral FWHM & $0.7 \lambda / 1.5 \lambda$ \\
& \\
Number of MBs per frame & Very low: $2 \pm 2$ \\
(mean \pm standard deviation (SD)) & Low: $8 \pm 4$ \\
& Medium: $28 \pm 13$ \\
& High: $49 \pm 20$ \\
Uncertainty in localization & Very low: $\lambda / 20$ \\
(SD of localization error) & Low: $\lambda / 10$ \\
& Medium: $\lambda / 5$ \\
& High: $\lambda / 2$ \\
\hline
\end{tabular}

\section{B. In vivo measurement}

The in vivo measurement was conducted using a modified BK5000 scanner, and an X18L5s transducer (BK Medical, 


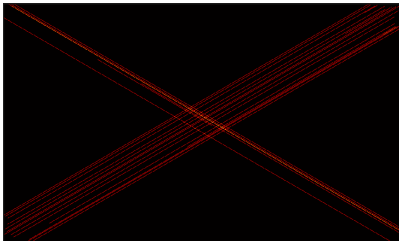

(a)

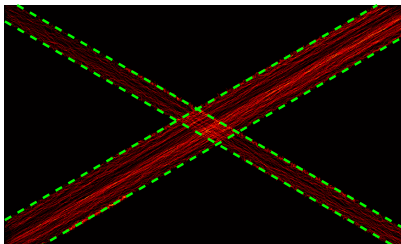

(e)

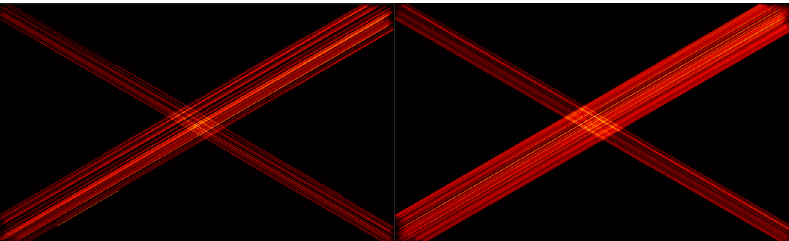

(b)

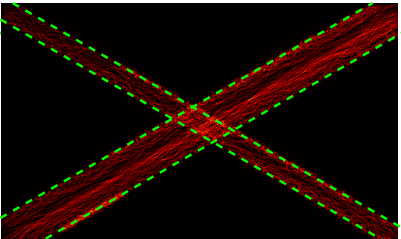

(f) (c)

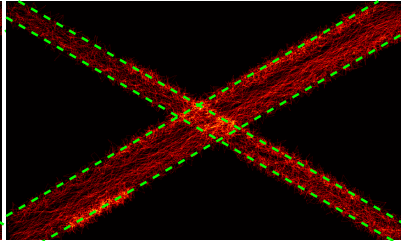

(g)

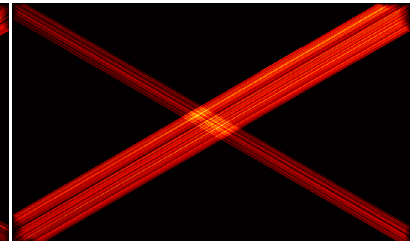

(d)

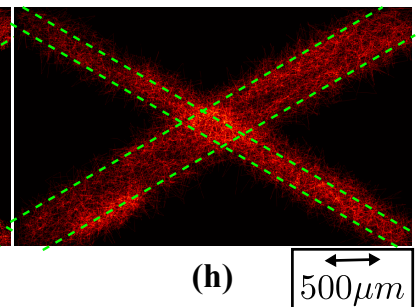

Fig. 1: Ground-truth tracks of various MB density scenarios for a (a) very low (b) low (c) medium or (d) high concentration. The tracks of uncertain MB positions with standard deviation of (e) $\lambda / 20$ (f) $\lambda / 10$ (g) $\lambda / 5$ and (h) $\lambda / 2$ for a low-density scenario are shown in the bottom row. The green dash lines show the wall of the tubes.

Herlev, Denmark) was used to record 10 minutes of contrastenhanced and B-mode frames from a rat kidney with a frame rate of $53.85 \mathrm{~Hz}$. A pulse amplitude modulation sequence was used for imaging with a transmit frequency of $6 \mathrm{MHz}(\lambda=$ $256 \mu \mathrm{m})$. The transmit voltage was low with a corresponding mechanical index (MI) of 0.2 to prevent bursting of the MBs. The MBs (SonoVue, Bracco, Milan, Italy) were injected with a flow-rate of $100 \mu \mathrm{l} / \mathrm{min}$ after a 1:10 dilution.

\section{Tracking algorithms}

In the NN tracker, the estimated MB positions in the next frame are linked to the $\mathrm{MB}$ positions in the current frame based on their minimum distance. Though NN is simple and fast, simple linking of MB positions generates false and flexuous tracks when the localization is uncertain. Considering the MB's smooth movement, more robust tracking is possible via Kalman filtering. This simple movement consideration can be modeled as $\vec{r}(t)=\vec{r}(t-1)+d \vec{r}(t)+\vec{\varepsilon}(t)$, where $\vec{r}(t)=\left(r_{z}(t), r_{x}(t)\right)$ is the position of $\mathrm{MB}$ at time $t$, $d \vec{r}(t)=\left(d r_{z}(t), d r_{x}(t)\right)$ is the displacement of the MB and $\varepsilon(t)$ is the uncertainty in the displacement. This model can be formulated within the Kalman framework as:

$$
\left\{\begin{array}{l}
\bar{x}(t)=\mathbf{F} x(t-1)+\varepsilon(t) \\
\bar{z}(t)=\mathbf{H} \bar{x}(t)+v(t),
\end{array}\right.
$$

where $x(t)=[\vec{r}(t), d \vec{r}(t)]^{T}=\left[r_{z}(t), r_{x}(t), d r_{z}(t), d r_{x}(t)\right]^{T}, \bar{x}(t)$ is the prediction of MB position in the next frame, $\bar{z}(t)$ is the estimated uncertain position,

$$
\mathbf{F}=\left[\begin{array}{llll}
1 & 0 & 1 & 0 \\
0 & 1 & 0 & 1 \\
0 & 0 & 1 & 0 \\
0 & 0 & 0 & 1
\end{array}\right], \quad \mathbf{H}=\left[\begin{array}{llll}
1 & 0 & 0 & 0 \\
0 & 1 & 0 & 0
\end{array}\right]
$$

$\varepsilon(t) \sim \mathscr{N}\left(0, \sigma_{\varepsilon}^{2}\right)$, and $v(t) \sim \mathscr{N}\left(0, \sigma_{v}^{2}\right)$.

In this study, the maximum linking distance was $500 \mu \mathrm{m}$, and the initial conditions of the Kalman filter were $\sigma_{\varepsilon}=1 \mathrm{~mm}$ and $\sigma_{v}=100 \mu \mathrm{m}$. After tracking, the velocity of the MBs was estimated by calculating the time derivative of track positions.

\section{Evaluation}

The first group of performance metrics, proposed in [12], was based on an assignment problem. To evaluate tracking performance using this approach, estimated tracks were paired with ground-truth tracks based on their minimum distance using Hungarian algorithm [13]. Hungarian algorithm provides fast optimal assignment of the two sets. The gate size is equal to $\lambda$, meaning that any tracks outside this gate range were considered as false positive (FP), tracks within the gate range were assumed as true positive (TP) or false negative $(\mathrm{FN})$, if they are paired or unpaired to the ground-truth. The Jaccard Similarity Coefficient (JSC) is defined as JSC = $\mathrm{TP} /(\mathrm{TP}+\mathrm{FP}+\mathrm{FN})$, which determines the similarity of the set of estimated tracks and set of ground-truth tracks. The root-meansquare error (RMSE) of velocity estimates was calculated for each paired track (TP), and the number of unpaired (spurious) tracks was also counted as an extra performance metric. For the paired tracks, JSC of track positions and RMSE of the estimated positions can also be calculated to provide metrics at the position level.

The second performance metric was calculated by considering the known velocity profile of flow in the tubes as a ground-truth. In this approach bias and standard deviation of velocity profiles over different cross sections of the tubes were calculated.

The localization uncertainty in the in vivo data, used as the last performance metric, was estimated by RMSE of a fitted piece-wise least-square line to the MB positions.

\section{RESULTS}

The super-resolution (SR) images shown in Fig. 2 indicate that the Kalman filter has more smooth tracks in this scenario. All of the assignment-based performance metrics for various density and uncertainty scenarios at the track and position levels are listed in Table II and Table III (the green labels show a better performance). At the position level, the Kalman filter outperformed the NN almost in all scenarios. Notice that the RMSE of the track positions are relevant to the localization 
TABLE II: Performance Metrics at Track Level (Color scheme: green $\rightarrow$ higher performance, yellow $\rightarrow$ roughly the same).

\begin{tabular}{|c|c|c|c|c|c|c|c|c|c|c|c|c|c|}
\hline \multirow{3}{*}{ MB Density } & \multirow{3}{*}{ Tracker } & \multicolumn{12}{|c|}{ Unertainty in Localization } \\
\hline & & \multicolumn{3}{|c|}{$\mathrm{SD}=\lambda / 20=12.83 \mu \mathrm{m}$} & \multicolumn{3}{|c|}{ SD $=\lambda / 10=25.67 \mu \mathrm{m}$} & \multicolumn{3}{|c|}{$\mathbf{S D}=\lambda / 5=51.33 \mu \mathrm{m}$} & \multicolumn{3}{|c|}{ SD $=\lambda / 2=128.34 \mu \mathrm{m}$} \\
\hline & & JSC & Spurious & RMSE & JSC & Spurious & RMSE & JSC & Spurious & RMSE & JSC & Spurious & RMSE \\
\hline \multirow{2}{*}{ Very low } & NN & 0.731 & 14 & 0.01 & 0.731 & 14 & 0.01 & 0.731 & 14 & 0.05 & 0.132 & 250 & 0.33 \\
\hline & Kalman & 1 & 0 & 0 & 1 & 0 & 0 & 1 & 0 & 0.03 & 0.528 & 34 & 0.01 \\
\hline \multirow{2}{*}{ Low } & NN & 0.961 & 13 & 3.19 & 0.953 & 16 & 0.01 & 0.835 & 64 & 3.22 & 0.148 & 1868 & 0.34 \\
\hline & Kalman & 0.934 & 23 & 0.03 & 0.923 & 27 & 0 & 0.91 & 32 & 0.01 & 0.416 & 454 & 0.03 \\
\hline \multirow{2}{*}{ Medium } & NN & 0.892 & 104 & 0 & 0.839 & 165 & 0.02 & 0.642 & 478 & 0.9 & 0.104 & 7437 & 0.17 \\
\hline & Kalman & 0.534 & 435 & 0.01 & 0.632 & 500 & 0.02 & 0.595 & 585 & 0.04 & 0.236 & 2194 & 0.19 \\
\hline \multirow{2}{*}{ High } & NN & 0.717 & 672 & 0.06 & 0.634 & 983 & 1.02 & 0.435 & 2209 & 0.3 & 0.095 & 16226 & 1.1 \\
\hline & Kalman & 0.446 & 2079 & 0.04 & 0.418 & 2376 & 0.04 & 0.388 & 2681 & 0.01 & 0.215 & 6209 & 0.9 \\
\hline
\end{tabular}

TABLE III: Performance Metrics at Position Level (Color scheme: green $\rightarrow$ higher performance, yellow $\rightarrow$ roughly the same).

\begin{tabular}{|c|c|c|c|c|c|c|c|c|c|}
\hline \multirow{3}{*}{ MB Density } & \multirow{3}{*}{ Tracker } & \multicolumn{8}{|c|}{ Unertainty in Localization } \\
\hline & & \multicolumn{2}{|c|}{$\mathbf{S D}=\lambda / \mathbf{2 0}$} & \multicolumn{2}{|c|}{$\mathbf{S D}=\lambda / \mathbf{1 0}$} & \multicolumn{2}{|c|}{$\mathbf{S D}=\lambda / \mathbf{5}$} & \multicolumn{2}{|c|}{$\mathbf{S D}=\lambda / 2$} \\
\hline & & JSC & RMSE & JSC & RMSE & JSC & RMSE & JSC & RMSE \\
\hline \multirow{2}{*}{ Very low } & $\mathbf{N N}$ & 0.639 & 15.02 & 0.639 & 29.49 & 0.576 & 52.27 & 0.127 & 135.23 \\
\hline & Kalman & 1 & 11.99 & 1 & 20.34 & 0.937 & 37.63 & 0.584 & 88.92 \\
\hline \multirow[b]{2}{*}{ Low } & NN & 0.754 & 14.3 & 0.672 & 25.12 & 0.557 & 38.86 & 0.154 & 115.25 \\
\hline & Kalman & 0.92 & 13.96 & 0.836 & 22.18 & 0.788 & 36.61 & 0.463 & 76.55 \\
\hline \multirow{2}{*}{ Medium } & NN & 0.511 & 15.54 & 0.432 & 15.58 & 0.354 & 29.21 & 0.094 & 112.72 \\
\hline & Kalman & 0.534 & 13.21 & 0.493 & 19.95 & 0.421 & 31.83 & 0.236 & 77.48 \\
\hline \multirow{2}{*}{ High } & NN & 0.446 & 16.52 & 0.36 & 22.62 & 0.266 & 40.09 & 0.078 & 111.92 \\
\hline & Kalman & 0.432 & 15.98 & 0.38 & 22.92 & 0.319 & 38.99 & 0.189 & 79.75 \\
\hline
\end{tabular}

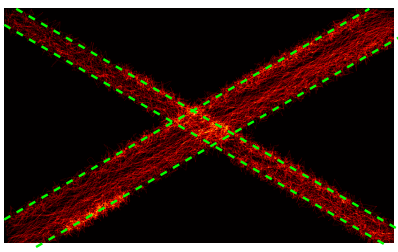

(a)

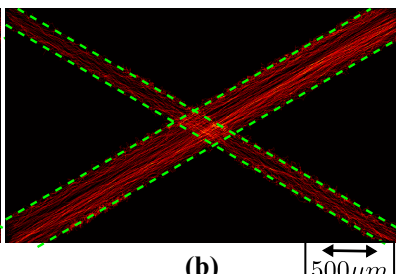

(b) $\quad 500 \mu \mathrm{m}$
Fig. 2: A comparison between (a) NN tracker, and (b) Kalman tracker for low-density scenario and uncertainty of $\lambda / 5$ in the localization.

uncertainty. This will be used later to estimate the uncertainty of localization from the in vivo measurements. At track level, NN achieved a better rank in JSC, and the number of spurious tracks was lower in several scenarios. However, the velocity estimates of paired tracks were more accurate when a Kalman filter was employed. This diverse behavior of trackers through different scenarios is discussed in Section IV. It can be seen that in some scenarios the Kalman filter can reduce the number of spurious tracks by more than $67 \%$.

To avoid the ambiguities in an assignment problem, the estimated velocity profiles over 14 cross-sections of the larger vessel in the final SR image are illustrated in Fig. 3. The velocity estimates and the resolution were improved by the Kalman filter. This shows the superiority of the Kalman over $\mathrm{NN}$ in a scenario with high MB concentration and uncertainty in the localization.

While there is no ground-truth for the in vivo measurement, less flexuous tracks are expected for more accurate localization or robust trackers. To measure the precision of the tracks positions, a piece-wise least-square line was fitted to MB positions tracked for more than 5 frames. Table IV lists the mean and SD of the localization error relative to the fitted line after NN and Kalman tracking. It shows that the Kalman filter has reduced the localization uncertainty. Fig. 4 shows SR images produced by the two trackers for 90 seconds of the in

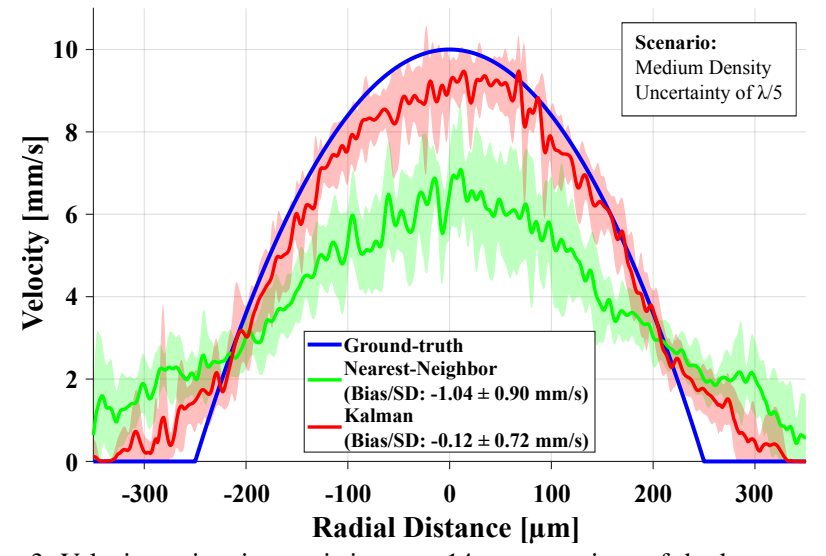

Fig. 3: Velocity estimation statistics over 14 cross-sections of the large vessel for medium density scenario with localization uncertainty of $\lambda / 5$. The NN estimates had a bias and standard deviation of $-1.04 \pm 0.9 \mathrm{~mm} / \mathrm{s}$, while these values for the Kalman estimates were reduced to $-0.12 \pm 0.72 \mathrm{~mm} / \mathrm{s}$.

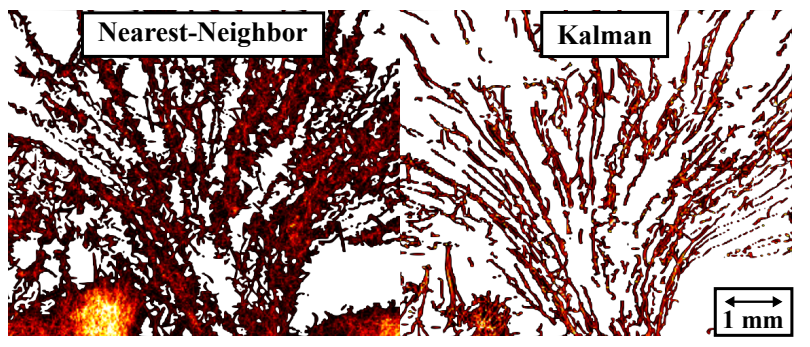

Fig. 4: Super-resolution images of renal inner medulla created using the NN (left) and Kalman (right) trackers. The NN generated a lot of spurious and flexous tracks. However, the Kalman tracker reduced these uncertainties. The structure is clearly distinguishable in the right image, as it is resolved down to $25.6 \mu \mathrm{m}$ for almost every position.

vivo data. It is clear that the Kalman tracker outperforms the $\mathrm{NN}$ tracker.

Finally, velocity SR images of the entire kidney, shown in Fig. 5, demonstrate the difference between two trackers in terms of less spurious and less uncertain tracks. 
TABLE IV: Tracking performance on localization uncertainty

\begin{tabular}{|c|c|}
\hline Tracker & Precision of individual track positions \\
\hline NN & $37.95 \pm 21.37 \mu \mathrm{m}$ \\
\hline Kalman & $23.9 \pm 11.82 \mu \mathrm{m}$ \\
\hline
\end{tabular}

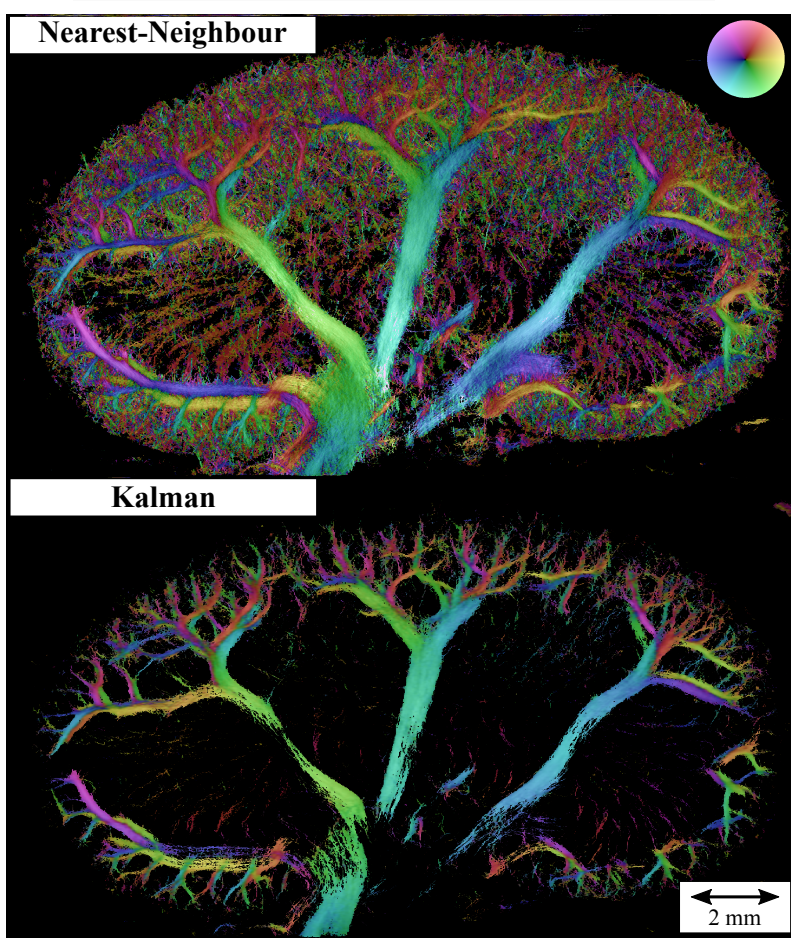

Fig. 5: Velocity SR images of the kidney show renal vascular tree with opposite flows (arteries and veins). The color wheel on top-right corner shows the flow direction of the color-coded tracks.

\section{DISCUSSION}

Considering only the JSC and the number of spurious tracks, NN performed better than Kalman in some scenarios. Two key factors explain this behavior of the Kalman tracker. Firstly, based on the definition of optimal assignment, a unique estimated track must be assigned to a ground-truth track. A tracker might estimate a long track with multiple small tracks. In this case, while tracks are correct, the number of spurious tracks will increase, and JSC will decrease. Therefore, these two metrics cannot determine the overall performance of the tracker in all scenarios. Secondly, a single Kalman filter with specific initial conditions is employed for tracking in all scenarios. A Kalman filter might not reach a fair estimate within a short lifetime of these MBs. The question that may arise here is why we do not optimize the tracker for each specific scenario? The reason is that in an in vivo measurement, the tracker has to deal with a wide range of scenarios, as the MBs have uncontrolled concentrations as well as different velocities and flow dynamics when entering the blood stream. All of these scenarios are usually difficult to track with a simple tracking strategy. One solution would be tracking with a hierarchical structure. For example, a Kalman filter with a strict initial condition can apply to track slow flows. Then, the untracked MBs and the track positions with an overestimated velocity can be passed to another Kalman filter optimized to track faster MBs. This can be continued in a hierarchical structure to cover all range of scenarios [14].

\section{CONCLUSION}

Several performance metrics for the tracking algorithms in SRI were investigated. It was shown that in the presence of a high localization uncertainty, the Kalman tracker reached a better velocity estimate bias and SD of $-0.12 \pm 0.72 \mathrm{~mm} / \mathrm{s}$ compared to $-1.04 \pm 0.9 \mathrm{~mm} / \mathrm{s}$ in the $\mathrm{NN}$ relative to a peak velocity of $10 \mathrm{~mm} / \mathrm{s}$. The Kalman tracker reduced the precision of individual track positions in an in vivo experiment to $37.95 \pm 21.37 \mu \mathrm{m}$ and $23.9 \pm 11.82 \mu \mathrm{m}$ for the $\mathrm{NN}$ and Kalman trackers. Overall, the Kalman tracker was superior, as it estimated velocity with a higher precision and reduced localization uncertainty.

\section{ACKNOWLEDGMENT}

This work was financially supported by grant 82-2014-4 from the Danish National Advanced Technology Foundation, by grant 7050-00004B from Innovation Fund Denmark, and from BK Medical, Herlev, Denmark.

\section{REFERENCES}

[1] O. M. Viessmann, R. J. Eckersley, K. Christensen-Jeffries, M. X. Tang, and C. Dunsby, "Acoustic super-resolution with ultrasound and microbubbles," Phys. Med. Biol., vol. 58, pp. 6447-6458, 2013.

[2] M. A. O'Reilly and K. Hynynen, "A super-resolution ultrasound method for brain vascular mapping," Med. Phys., vol. 40, no. 11, pp. 110701-7, 2013.

[3] Y. Desailly, J. Pierre, O. Couture, and M. Tanter, "Resolution limits of ultrafast ultrasound localization microscopy," Phys. Med. Biol., vol. 60 , no. 22, pp. 8723-8740, 2015.

[4] K. Christensen-Jeffries, R. J. Browning, M. Tang, C. Dunsby, and R. J. Eckersley, "In vivo acoustic super-resolution and super-resolved velocity mapping using microbubbles," IEEE Trans. Med. Imag., vol. 34, no. 2, pp. 433-440, February 2015.

[5] C. Errico, J. Pierre, S. Pezet, Y. Desailly, Z. Lenkei, O. Couture, and M. Tanter, "Ultrafast ultrasound localization microscopy for deep superresolution vascular imaging," Nature, vol. 527, pp. 499-502, November 2015.

[6] D. Ackermann and G. Schmitz, "Detection and tracking of multiple microbubbles in ultrasound B-mode images," IEEE Trans. Ultrason., Ferroelec., Freq. Contr., vol. 63, no. 1, pp. 72-82, January 2016.

[7] K. Christensen-Jeffries, O. Couture, P. A. Dayton, Y. C. Eldar, K. Hynynen, F. Kiessling, M. O'Reilly, G. F. Pinton, G. Schmitz, M. Tang et al., "Super-resolution ultrasound imaging," Ultrasound Med. Biol., vol. 46, no. 4, pp. 865-891, 2020.

[8] J. C. Crocker and D. G. Grier, "Methods of digital video microscopy for colloidal studies," J. Colloid, vol. 179, no. 1, pp. 298-310, 1996.

[9] O. Solomon, R. J. G. van Sloun, H. Wijkstra, M. Mischi, and Y. C. Eldar, "Exploiting flow dynamics for super-resolution in contrast-enhanced ultrasound," IEEE Trans. Ultrason., Ferroelec., Freq. Contr., vol. 60, no. 10 , pp. 1573-1586, 2019.

[10] S. Tang, P. Song, J. D. Trzasko, M. Lowerison, C. Huang, P. Gong, U. Lok, A. Manduca, and S. Chen, "Kalman filter-based microbubble tracking for robust super-resolution ultrasound microvessel imaging," IEEE Trans. Ultrason., Ferroelec., Freq. Contr., vol. PP, no. 99, pp. $1-1,2020$.

[11] W. J. Godinez, M. Lampe, S. Worz, B. Muller, R. Eils, and K. Rohr, "Deterministic and probabilistic approaches for tracking virus particles in time-lapse fluorescence microscopy image sequences," Med. Image Anal., vol. 13, no. 2, pp. 325-342, 2009.

[12] N. Chenouard, I. Smal, F. D. Chaumont, M. Maška, I. F. Sbalzarini, Y. Gong, J. Cardinale, C. Carthel, S. Coraluppi, M. Winter et al., "Objective comparison of particle tracking methods," Nature methods, vol. 11, no. 3, pp. 281-290, 2014.

[13] H. W. Kuhn, "The Hungarian method for the assignment problem," Naval research logistics quarterly, vol. 2, no. 1-2, pp. 83-97, 1955.

[14] I. Taghavi et al., "In vivo ultrasound super-resolution imaging with motion correction and robust tracking," IEEE Trans. Med. Imag., p. submitted, 2020 . 